\title{
THE $K$-SPAN OF A RIEMANN SURFACE
}

\author{
BY MYRON GOLDSTEIN
}

\author{
Communicated by F. W. Gehring, May 11, 1970
}

In this note, we shall answer a question first posed by Sario and Oikawa in their monograph Capacity functions [4], and later by Rodin [3] in his Bulletin paper. The question is this. Does the class $K D$ of harmonic functions $u$ with finite Dirichlet integral and such that $* d u$ has vanishing periods along all dividing cycles consist only of constant functions if and only if the $K$-span (for $m=0$ ) vanishes at some point with respect to some local parameter about it? The $K$-span (for $m=0$ ) is defined to be $\partial v /\left.\partial x\right|_{z m \xi}$ where $d v$ reproduces for the space $d K D$, i.e. $(d u, d v)=\pi \partial u /\left.\partial x\right|_{z=5}$ for all $d u \in d K D$, and $z$ denotes a local variable at $\zeta$. Note that the definition of the span depends on $\zeta$ and the choice of the local variable at $\zeta$.

We shall answer this question in the negative by exhibiting a Riemann surface which carries nonconstant $K D$ functions but which has the property that the $K$-span (for $m=0$ ) vanishes at each point for some choice of the local variable at that point. Note that our $K$-span (for $m=0$ ) is Rodin's 1-span.

The Riemann surface we shall construct is the same one that appears on p. 377 of my paper Boundaries of function spaces of Riemann surfaces [2], but, in order to aid the reader, the details of the construction will be repeated here.

Let $R_{0}$ be a hyperbolic Riemann surface which admits no nonconstant harmonic functions with finite Dirichlet integral and has a single ideal boundary component. Let $\left\{\gamma_{n}\right\}$ denote a sequence of analytic Jordan arcs on $R_{0}$ such that $\gamma_{n} \cap \gamma_{m}=\varnothing$ for $n \neq m$, and such that for an arbitrary compact subset $K$ of $R_{0}, \gamma_{n} \cap K=\varnothing$ for all sufficiently large $n$. Let $R^{\prime}=R_{0}-\cup_{n=1}^{\infty} \gamma_{n}$ and take the sequence $\left\{\gamma_{n}\right\}$ such that $R^{\prime}$ does not belong to the class $S O_{H D^{\prime}}$ i.e. such that there exists a nonnegative Dirichlet function on $R_{0}$ which is harmonic on $R^{\prime}$ and vanishes quasi everywhere on $\bigcup_{n=1}^{\infty} \gamma_{n}$ but does not vanish quasi everywhere on $R_{0}$. Let $R_{1}^{\prime}$ and $R_{2}^{\prime}$ be two copies of $R^{\prime}$. Denote by $\gamma_{n}^{+}$(resp. $\gamma_{n}^{-}$) the positive (resp. negative) edge of $\gamma_{n}$. For each $n$, identify $\gamma_{n}^{+}$of $R_{1}^{\prime}$ with $\gamma_{n}^{-}$of $R_{2}^{\prime}$ and $\gamma_{n}^{-}$of $R_{1}^{\prime}$ with $\gamma_{n}^{+}$of $R_{2}^{\prime}$. The resulting Riemann surface $R$ has a single ideal boundary component. Furthermore, $R \in 0_{H D}^{2}-0_{H D}^{1}$, i.e. the dimension of the vector

AMS 1970 subject classifications. Primary 3045; Secondary 3111.

Key words and phrases. $H D$ functions, $K D$ functions, level curve, critical point, span, ideal boundary component. 
lattice $H D$ of harmonic functions on $R$ with finite Dirichlet integral is 2 . Since $R$ has a single ideal boundary component, $K D=H D$, and hence $R$ possesses nonconstant $K D$ functions. Let $\zeta$ be a point of $R$ and $u$ a nonconstant $K D$ function on $R$. Consider the level curve $u=u(\zeta)$. If $\zeta$ is a critical point of $u$, then since $K D$ is two dimensional, $\zeta$ will be a critical point of every $K D$ function and hence the $K$-span (for $m=0$ ) will vanish at $\zeta$ for every choice of the local variable at $\zeta$. If $\zeta$ is not a critical point of $u$, then a portion of the level curve $u=u(\zeta)$ passing through $\zeta$ will be an analytic Jordan arc $\gamma$. Hence there exists a parametric disk $(V, \phi)$ about $\zeta$ such that $\phi(\gamma)$ $=\{z:-1<x<1, y=0\}$. Since $u$ is constant along $\phi(\gamma)$, it follows that $\partial u / \partial x$ vanishes at $\zeta$. If $d v$ denotes the reproducing kernel for the space $d K D$ at $\zeta$, the choice of the local variable at $\zeta$ will be that determined by $\phi$, it follows that $\partial v /\left.\partial x\right|_{z=\zeta}=0$, i.e. the $K$-span (for $m=0$ ) vanishes at $\zeta$, since $K D$ is two dimensional.

It is interesting to note that every $K D$ function $u$ takes the same value at each of the branch points. To see this, let $\sigma: R \rightarrow R$ denote the natural involution map of $R$ onto itself, i.e. if $\zeta^{\prime}$ and $\zeta^{\prime \prime}$ lie over the same point $\zeta$ of $R_{0}$, then $\sigma\left(\zeta^{\prime}\right)=\sigma\left(\zeta^{\prime \prime}\right)$. Since $R_{0}$ admits no nonconstant $H D$ functions, it follows that $u+u \circ \sigma=c$ where $c$ is a constant. Hence if $P$ is a branch point of $R, u(P)=c / 2$ for all $K D$ functions $u$.

\section{REFERENCES}

1. L. V. Ahlfors and L. Sario, Riemann surfaces, Princeton Math. Series, no. 26, Princeton Univ. Press, Princeton, N. J., 1960. MR 22 \#5729.

2. M. Goldstein, Boundaries of function spaces of Riemann surfaces, Math. Z. 110 (1969), 375-377.

3. B. Rodin, On the span of a Riemann surface, Bull. Amer. Math. Soc. 76 (1970), 340-341.

4. L. Sario and K. Oikawa, Capacity functions, Die Grundlehren der math. Wissenschaften, Band 149, Springer-Verlag, Berlin and New York, 1969.

Oregon State University, Corvallis, Oregon 97331 